\title{
A Modified Particle Swarm Optimization Algorithm
}

\author{
Ai-Qin $\mathrm{Mu}^{1,2}$, De-Xin Cao ${ }^{1}$, Xiao-Hua Wang ${ }^{2}$ \\ ${ }^{1}$ College of Science, China University of Mining \& Technology, XuZhou, China; muaqin@126.com, caodx@cumt.edu.cn \\ ${ }^{2}$ Foundation Departments, Xuzhou Air Force Academy, XuZhou, China
}

Received 17 August 2009; revised 28 August 2009; accepted 30 August 2009.

\begin{abstract}
Particle Swarm Optimization (PSO) is a new optimization algorithm, which is applied in many fields widely. But the original PSO is likely to cause the local optimization with premature convergence phenomenon. By using the idea of simulated annealing algorithm, we propose a modified algorithm which makes the most optimal particle of every time of iteration evolving continuously, and assign the worst particle with a new value to increase its disturbance. By the testing of three classic testing functions, we conclude the modified PSO algorithm has the better performance of convergence and global searching than the original PSO.
\end{abstract}

Keywords: PSO; Simulated Annealing Algorithm; Global Searching

\section{INTRODUCTION}

PSO algorithm is a new intelligent optimization algorithm intimating the bird swarm behaviors, which was proposed by psychologist Kennedy and Dr. Eberhart in 1995 [1]. Compared with other optimization algorithms, the PSO is more objective and easily to perform well, it is applied in many fields such as the function optimization, the neural network training, the fuzzy system control, etc.

In PSO algorithm, each individual is called "particle", which represents a potential solution. The algorithm achieves the best solution by the variability of some particles in the tracing space. The particles search in the solution space following the best particle by changing their positions and the fitness frequently, the flying direction and velocity are determined by the objective function.

For improving the convergence performance of PSO, the inertia factor $w$ is used by Shi and Eberhart [2] to control the impact on current particle by former particle's velocity. PSO algorithm has preferred global searching ability when $w$ is relatively large. On the contrary, its local searching ability becomes better when $w$ is smaller. Now the PSO algorithm with inertia weight factor was called standard PSO.

However, in PSO algorithm, particles would lost the ability to explore new domains when they are searching in solution space, that is to say it will entrap in local optimization and causes the premature phenomenon. Therefore, it is very import for PSO algorithm to be guaranteed to converge to the global optimal solution, and many modify PSO algorithms were researched in recent ten years. For example, linearly decreasing inertia weight technique was studied in [3].

In order to solve the premature phenomenon, many modified algorithms based on Simulated Annealing Algorithm are proposed. For example, the new location of all particles is selected according to the probability $[4,5]$; the PSO and simulated annealing algorithm are iterated alternatively [6,7]; Gao Ying and Xie Shengli [8] add hybridization and Gaussian mutation to alternative iterations; in [9] particles are divided into two groups, PSO and simulated annealing algorithm are iterated to them respectively and then mixed two algorithms. This paper proposed a new modify PSO algorithm. The arrangement of this paper is as follows. In section 2, the principle of standard PSO is introduced. In section 3, the modified PSO algorithm is described. In section 4, three benchmark functions are used to evaluate the performance of algorithm, and the conclusions are given in section 5 .

\section{STANDARD PSO ALGORITHM}

Assuming $X_{i}=\left(x_{i 1}, x_{i 2}, \cdots, x_{i D}\right)$ is the position of $i$-th particle in D-dimension, $V_{i}=\left(v_{i 1}, v_{i 2}, \cdots, v_{i D}\right)$ is its velocity which represents its direction of searching. In iteration process, each particle keeps the best position pbest found by itself, besides, it also knows the best position gbest searched by the group particles, and changes its velocity according two best positions. The standard 
formula of PSO is as follow:

$$
\begin{gathered}
v_{i d}{ }^{k+1}=w v_{i d}{ }^{k}+c_{1} r_{1}\left(p_{i d}-x_{i d}{ }^{k}\right)+c_{2} r_{2}\left(p_{g d}-x_{i d}{ }^{k}\right) \\
x_{i d}{ }^{k+1}=x_{i d}{ }^{k}+v_{i d}{ }^{k+1}
\end{gathered}
$$

In which: $i=1,2, \cdots N ; N$-the population of the group particles; $d=1,2, \cdots, D ; k$-the maximum number of iteration; $r_{1}, r_{2}$-the random values between $[0,1]$, which are used to keep the diversity of the group particles; $c_{1}, c_{2}$-the learning coefficients, also are called acceleration coefficients; $v_{i d}{ }^{k}$-the number $\mathrm{d}$ component of the velocity of particle $i$ in $k$-th iterating; $x_{i d}{ }^{k}$-the number $\mathrm{d}$ component of the position of particle $i$ in $k$-th iterating; $p_{i d}$-the number $\mathrm{d}$ component of the best position particle $\mathrm{i}$ has ever found; $p_{g d}$-the number $\mathrm{d}$ component of the best position the group particles have ever found.

The procedure of standard PSO is as following:

1) Initialize the original position and velocity of particle swarm;

2) Calculate the fitness value of each particle;

3) For each particle, compare the fitness value with the fitness value of pbest, if current value is better, then renew the position with current position, and update the fitness value simultaneously;

4) Determine the best particle of group with the best fitness value, if the fitness value is better than the fitness value of gbest, then update the gbest and its fitness value with the position;

5) Check the finalizing criterion, if it has been satisfied, quit the iteration; otherwise, return to step 2).

\section{THE MODIFIED PSO}

In standard PSO, because the particle has the ability to know the best position of the group particles have been searched, we need one particle to find the global best position rather than all particles to find it, and other particles should search more domains to make sure the best position is global best position not the local one. Based on these ideas, we propose some modifications with the standard PSO algorithm. Firstly, the modified algorithm chooses the particle with maximum fitness when it is iterating, initializes its position randomly for increasing the chaos ability of particles. By this means, the particle can search more domains. Secondly, by referring to ideas of the simulated annealing algorithm and using neighborhoods to achieve the guaranteed convergence PSO in [10], it is hoped that the fitness of the particle which has the best value in last iteration would be smaller than last times, and it is acceptable the fitness is worse in a limited extent $\alpha$. We calculate the change of fitness value of two positions $\Delta f$, and accept the new position if $\Delta f$ is smaller than $\alpha$. Otherwise, a new position is assigned to the particle randomly from its neighborhood with radius $r$.

The procedure of modified PSO is as following:

1) Initialize the position and velocity of each particle;

2) Calculate the fitness of each particle;

3) Concern the particle with the biggest fitness value, reinitialize its position; and evaluate the particle with the smallest fitness value whether its new position is acceptable, if the answer is yes, update its position, otherwise, a new position is assigned to the particle randomly in its neighborhood with radius $r$; then renew the position and velocity of other particles according to Formula (1) and (2);

4) For each particle, compare its current fitness value with the fitness of its pbest, if the current value is better, then update pbest and its fitness value;

5) Determine the best particle of group with the best fitness value, if the current fitness value is better than the fitness value of gbest, then update the gbest and its fitness value with the position;

6) Check the finalizing criterion, if it has been satisfied, quit the iteration; otherwise, return to step 3).

\section{NUMERICAL SIMULATION}

For investigating the modified PSO's convergence and searching performance, three benchmark functions are used to compare with standard PSO in this section. The basic information of three functions is described in Table 1.

Benchmark function 1 is non-linear single-peak function. It is relatively simple, and mainly used to test the accuracy of searching optimization.

Table 1. Benchmark functions used in experiment.

\begin{tabular}{ccc}
\hline expression & minimum point & optimal solution \\
\hline$F_{1}=\left(x_{1}-x_{2}\right)^{2}+\left(\left(x_{1}+x_{2}-10\right) / 3\right)^{2}$ & $(5,5)$ & 0 \\
$F_{2}=100\left(x_{2}-x_{1}{ }^{2}\right)^{2}+\left(1-x_{1}\right)^{2}$ & $(1,1)$ & 0 \\
$F_{3}=\sum_{i=1}^{2}\left[x_{i}^{2}-10 \cos \left(2 \pi x_{i}\right)+10\right]$ & $(1,1)$ & 0 \\
\hline
\end{tabular}


Table 2. Results of experiment.

\begin{tabular}{cccccc}
\hline $\begin{array}{c}\text { Benchmark } \\
\text { function }\end{array}$ & Algorithm & $\begin{array}{c}\text { Total number of } \\
\text { iterations }\end{array}$ & $\begin{array}{c}\text { Mean of optimal } \\
\text { solution }\end{array}$ & $\begin{array}{c}\text { Minimum of optimal } \\
\text { solution }\end{array}$ & $\begin{array}{c}\text { Minimum times of } \\
\text { iteration }\end{array}$ \\
\hline \multirow{2}{*}{$F_{1}$} & standard PSO & 20795 & $4.3708 \mathrm{e}-6$ & $6.9446 \mathrm{e}-9$ & 130 \\
& modified PSO & 10595 & $2.1498 \mathrm{e}-6$ & $1.26 \mathrm{e}-9$ & 68 \\
$F_{2}$ & standard PSO & 23836 & $1.7674 \mathrm{e}-4$ & $1.9403 \mathrm{e}-8$ & 205 \\
& modified PSO & 21989 & $8.8366 \mathrm{e}-6$ & $2.52012 \mathrm{e}-8$ & 350 \\
$F_{3}$ & standard PSO & 24990 & 0.0667 & $8.9467 \mathrm{e}-8$ & 237 \\
\hline
\end{tabular}

Benchmark function 2 is typical pathological quadratic function which is difficult to be minimized. There is a narrow valley between its global optimum and the reachable local optimum, the chance of finding the global optimal point is hardly. It is typically used to evaluate the implementation of the performance optimization.

Base on sphere function, benchmark function 3 uses cosine function to produce a mounts of local minimum, it is a typical complex multi-peak function which has the massive local optimal point. This function is very easy to make the algorithm into a local optimum not the global optimal solution.

In experiment, the population of group particle is 40 ; $c_{1}$ and $c_{2}$ are set to 2 ; the maximum time of iteration is 10000. It is acceptable if the difference between the best solution obtained by the optimization algorithm and the true solution is less then 1e-6. In standard PSO and modified PSO, the inertia weight is linear decreasing inertia all, which is determined by the following equation:

$$
w=w_{\text {max }}-\frac{w_{\text {max }}-w_{\text {min }}}{i t e r_{\text {max }}} \times k
$$

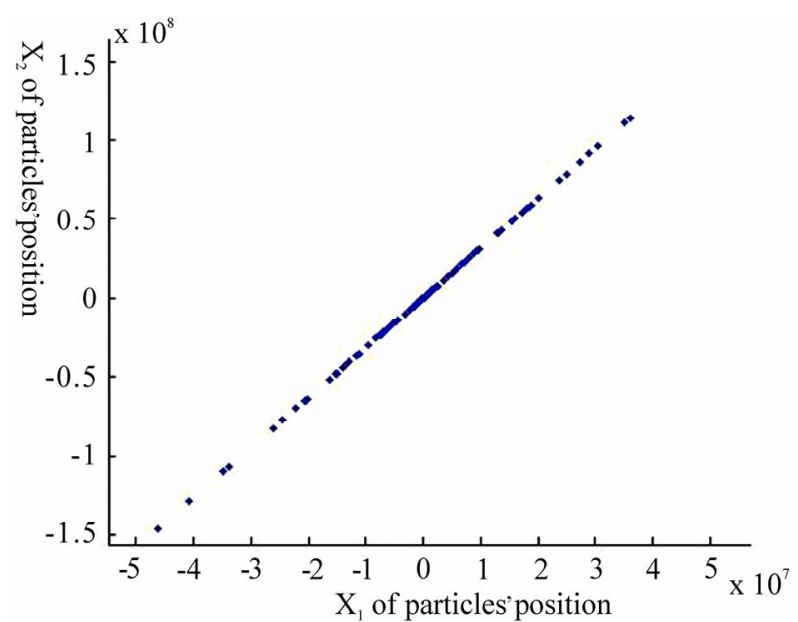

Figure 1. Path of standard PSO's particle.
Where $w_{\max }$ is the start of inertia weight which is set to 0.9 , and $w_{\min }$, the end of inertia weight, is set to 0.05 . iter $_{\max }$ is the maximum times of iteration; $k$ is the current iteration times. In order to reflect the equity of experiment, two algorithms all use the same original position and velocity randomly generated.

Parameter $\alpha$ which represents the acceptable limited extent of the fitness value is set to 0.5 in modified PSO. For using less parameter, the dynamic neighborhood is used and its radius is set to $w$. Each experiment is Executed 30 times, and takes their total iteration times and mean optimal solution for comparing. Table 2 presents the results of experiment.

From Table 2, it is easy to find that the modified PSO takes half time as standard PSO to achieve the best solution of function 1. Although the modified PSO has not remarkable improvement in convergence rate from function 2, its mean optimal solution is better than standard PSO, which implies the modified PSO has the better performance in global searching, the conclusion is proved in function 3 . Though the total number of iteration of standard PSO is less than modified PSO, but its mean optimal solution is 0.0667 , that indicate the rapid convergence of standard PSO is built on running into local optimal. On the contrary, the modified PSO can jump from local optimal successfully, that enhances the stability of algorithm greatly. Observe the results of modified PSO concretely; it can be found that the worst optimal solution of all iterations is $8.5442 \mathrm{e}-5$, which indicates the convergence rate is $100 \%$. The details of 30 loops will no longer run them out.

For observing the movement of particles from benchmark function 3, the standard PSO and the modified PSO are run again by giving the same position and velocity of each particle initialized randomly. The result is that standard PSO has iterated 800 times for the optimal solution to be $1.3781 \mathrm{e}-6$, and the modified PSO has iterated 1216 times for the optimal solution to be 6.3346e-7. A particle is randomly chosen to observe its trace. Figure 1 and Figure 2 present the result. 


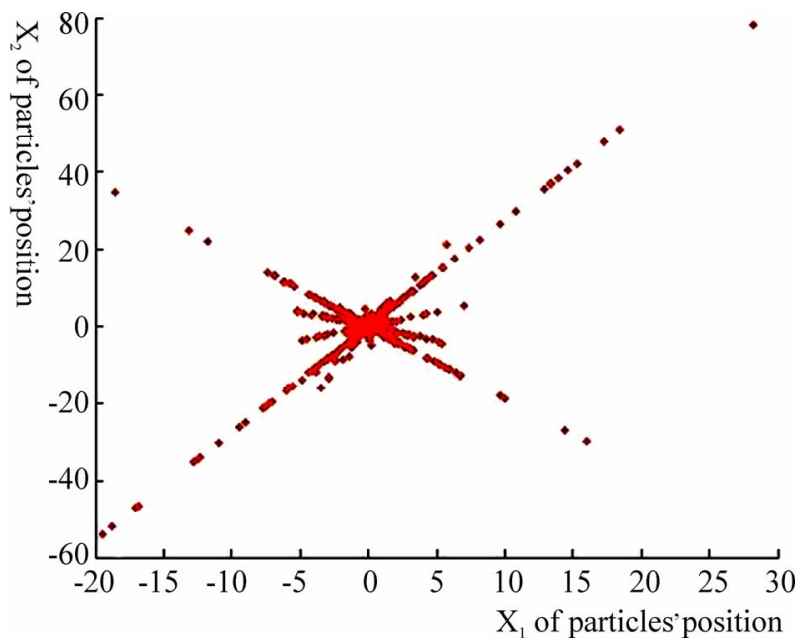

Figure 2. Path of modified PSO's particle.

From Figure 1 and Figure 2, it is easily to find out that the particle of standard PSO was vibrating nearby the optimal position until converging at the optimal position, otherwise, the particle of modified PSO has searched more domains then jumped from the local optimal solution, which ensured the algorithm to converge to the global optimal solution stably.

Generally, the improvement of the modified PSO based on simulated annealing algorithm is applied to all particles. In order to compare the performance of algorithms, we proposed the second improvement to all particles based on the ideas that mentioned before in this article, the main idea is that it is acceptable for all parti- cles when their fitness would be worse in a limited extent $\alpha$ at the next iteration, otherwise, new positions are assigned to the particles randomly from their neighborhood with radius $r$.

Next, the total iteration times and mean optimal solution are compared between modified PSO and the second improvement from three benchmark functions. The parameters are set as following: the original velocity is 0 ; other parameters are just same as former case. The experiment is executed 100 times and same original settings are assigned randomly. The results are shown in Table 3. If the maximum and minimum velocity of particles are limited to 1 and -1 , Table 4 shows the results.

From Table 3 and Table 4, it is obviously that both modified PSO and the second improvement can jump from local optimal convergence, which means they have the better global searching performance. For function 2 and function 3, the convergence rate of modified PSO is faster than the second improvement. It implied that although the modified PSO do some modifications to two particles' movement, the results is not worse than do the same modifications to all particles', sometimes, it has the better convergence performance. For function 1, when the velocity of particle is not limited, the performance of modified PSO can not compare with the second improvement, but they have the same performance when $V_{\max }$ and $V_{\min }$ are limited. If compare vertically, it can be found that whether the modified PSO or the second improvement have better convergence rate when the velocity of particles is limited. Especially, the second improvement has half times iteration of the modified

Table 3. Performance comparison between modified PSO and the second improvement without limited velocity.

\begin{tabular}{|c|c|c|c|c|}
\hline \multirow[t]{2}{*}{ benchmark function } & \multicolumn{2}{|c|}{ total iteration times } & \multicolumn{2}{|c|}{ mean optimal solution } \\
\hline & modified PSO & second improvement & modified PSO & second improvement \\
\hline$F_{1}$ & 33036 & 13063 & $4.3640 \mathrm{e}-6$ & $1.5878 \mathrm{e}-6$ \\
\hline$F_{2}$ & 72438 & 86025 & $1.3661 \mathrm{e}-5$ & $2.3398 \mathrm{e}-5$ \\
\hline$F_{3}$ & 93506 & 155573 & $1.1128 \mathrm{e}-5$ & $2.7690 \mathrm{e}-4$ \\
\hline
\end{tabular}

Table 4. Performance comparison between modified PSO and the second improvement with limited velocity.

\begin{tabular}{|c|c|c|c|c|}
\hline \multirow[t]{2}{*}{ benchmark function } & \multicolumn{2}{|c|}{ total iteration times } & \multicolumn{2}{|c|}{ mean optimal solution } \\
\hline & modified PSO & second improvement & modified PSO & second improvement \\
\hline$F_{1}$ & 12381 & 12034 & $1.7625 \mathrm{e}-6$ & $9.1580 \mathrm{e}-7$ \\
\hline$F_{2}$ & 37917 & 60139 & $8.1302 \mathrm{e}-6$ & $9.1149 \mathrm{e}-5$ \\
\hline$F_{3}$ & 53453 & 131291 & $1.3804 \mathrm{e}-5$ & $2.4989 \mathrm{e}-4$ \\
\hline
\end{tabular}


PSO. This proved that it is important for PSO to limit the velocity of particles.

\section{CONCLUSIONS}

In this paper, a modified PSO is proposed based on the simulated annealing algorithm. Through the results achieved in experiments, we can draw following conclusions:

1) The modified PSO has a better performance in stability and global convergence; it is the most important conclusion.

2) Although the modified PSO do some modifications to two particles' position and velocity, but its convergence rate for the multi-peak function is much faster as compared with the second improvement.

3) In modified PSO, the maximum and minimum velocity of particles have obvious impact on the convergence rate. How to choose the appropriate velocity limitation is the next step in our research.

\section{REFERENCES}

[1] Kennedy, J. and Eberhart, R.C. (1995) P article swarm optimization. IEEE International Conference on Neural Network, 1942-1948.

[2] Shi, Y. and Eberhart, R.C. (1998) A modified particle swarm optimizer. Proceedings of Congress on Evolutionary Computation, 79-73.
[3] Shi, Y. and Eberhart, R.C. (1999) Empirical study of particle swarm optimization. Proceedings of the 1999 Congress on Evolutionary Computation, 1945-1950.

[4] Wang, L.Z. (2006) Optimization of the solution to the problems simulated annealing to improve particle swarm algorithm. Journal of Liuzhou Teachers College, 21(3), 101-103.

[5] Gao, S., Yang, J.Y., Wu, X.J. and Liu, T.M. (2005) Particle swarm optimization based on the ideal of simulated annealing algorithm. Computer Applications and Software, 22(1), 103-104.

[6] Wang, Z.S., Li, L.C. and Li, B. (2008) Reactive power optimization based on particle swarm optimization and simulated annealing cooperative algorithm. Journal of Shandong University (Engineering Science), 38(6), 15-20.

[7] Wang, L.G., Hong, Y., Zhao, F.Q. and Yu, D.M. (2008) A hybrid algorithm of simulated annealing and particle swarm optimization. Computer Simulation, 25(11), 179182.

[8] Gao, Y. and Xie, S.L. (2004) Particle swarm optimization algorithms based on simulated annealing. Computer Engineering and Applications, 40(1), 47-50.

[9] Pan, Q.K., Wang, W.H. and Zhu, J.Y. (2006) Effective hybrid heuristics based on particle swarm optimization and simulated annealing algorithm for job shop scheduling. Chinese Journal of Mechanical Engineering, 17(10), 1044-1046.

[10] Peer, E.S., Van den Bergh, F. and Engelbrecht A.P. (2003) Using neighbourhoods with the guaranteed convergence PSO. Proceeding of the IEEE Swarm Intelligence Symposium, 235-242. 